\title{
Supplement to: Silicon isotope fractionation and uptake dynamics of three crop plants: laboratory studies with transient silicon concentrations
}

Daniel A. Frick ${ }^{1}$, Rainer Remus ${ }^{2}$, Michael Sommer ${ }^{2,3}$, Jürgen Augustin ${ }^{2}$, Friedhelm von Blanckenburg ${ }^{1,4}$

$5 \quad{ }^{1}$ GFZ German Research Centre for Geosciences, Potsdam, 14473, Germany.

${ }^{2}$ Leibniz Centre for Agricultural Landscape Research (ZALF), Müncheberg, 15374, Germany.

${ }^{3}$ Institute of Environmental Science and Geography, University of Potsdam, Potsdam, 14476, Germany

${ }^{4}$ Institute of Geological Science, Freie Universität Berlin, Berlin, 12249, Germany.

Correspondence to: Daniel A. Frick (dfrick@gfz-potsdam.de) 

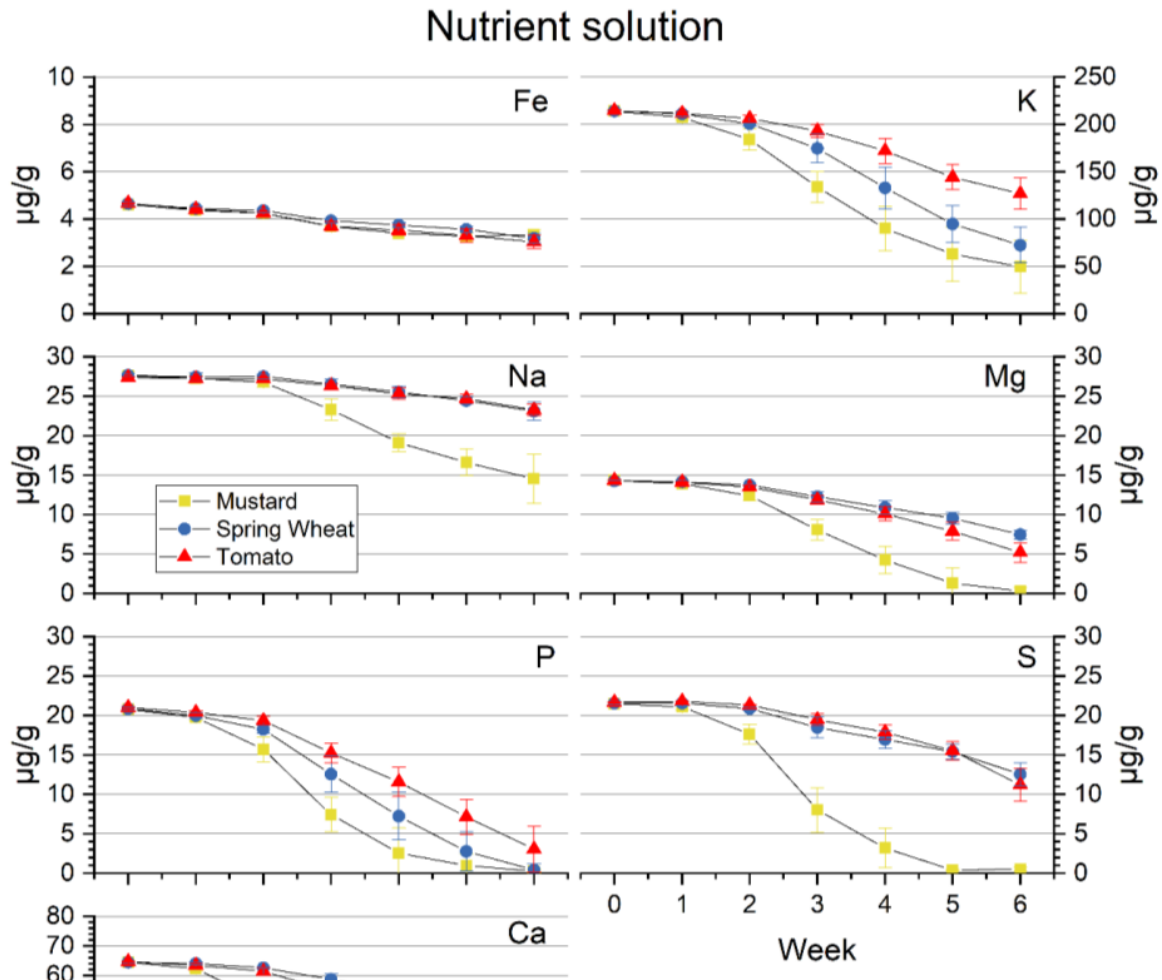
Concentration ( $\mathrm{Fe}, \mathrm{K}, \mathrm{Mg}, \mathrm{Ca}, \mathrm{P}, \mathrm{S}, \mathrm{Na}$ in $\mu \mathrm{g} \cdot \mathrm{g}^{-1}$ ) are based on the mean of the three replicated containers, uncertainty shown is 1 standard deviation of those replicated containers. 
Tables

\begin{tabular}{|c|c|c|}
\hline & Concentration measurements & Si isotope ratio measurements \\
\hline Instrument & Varian 720ES ICP-OES & ThermoFisher Neptune Plus \\
\hline Spraychamber & cyclonic, glass & APEX \\
\hline Nebuliser & concentric, glass & concentric, PFA \\
\hline Sample uptake rate & ca. $2 \mathrm{ml} / \mathrm{min}$ (pumped: $15 \mathrm{rpm}$ ) & $160 \mu \mathrm{L} / \mathrm{min}$ \\
\hline Cones & standard cone & N-sampler / H-skimmer \\
\hline Plasma RF power & $1.0 \mathrm{~kW}$ & $1200 \mathrm{~W}$ \\
\hline Ar cool gas & $15 \mathrm{~L} / \mathrm{min}$ & $15 \mathrm{~L} / \mathrm{min}$ \\
\hline Ar aux gas & $1.5 \mathrm{~L} / \mathrm{min}$ & $0.8 \mathrm{~L} / \mathrm{min}$ \\
\hline $\begin{array}{l}\text { Ar nebuliser pressure /flow } \\
\text { rate }^{\mathrm{a}}\end{array}$ & $280-320 \mathrm{kPa}$ & $1.0 \mathrm{~L} / \mathrm{min}$ \\
\hline Analysis integration time & $10 \mathrm{~s}$ & $4 \mathrm{~s}$ \\
\hline $\begin{array}{l}\text { Integration replicates } \\
\text { analysis }\end{array}$ & 3 & 30 \\
\hline Rinse time between samples & $60 \mathrm{~s}$ (pumped at $50 \mathrm{rpm}), 0.3 \mathrm{M} \mathrm{HNO}_{3}$ & $160 \mathrm{~s}, 0.1 \mathrm{M} \mathrm{HCl}$ \\
\hline $\begin{array}{l}\text { Analytes (wavelengths in } \mathrm{nm} \\
\text { for ICP-OES or isotopes for } \\
\text { MC-ICP-MS) }\end{array}$ & $\begin{array}{l}\mathrm{Ca} 422.673, \mathrm{Fe} 238.204, \mathrm{~K} 769.897, \mathrm{Mg} \\
\text { 280.270, Na 588.995, Si } 288.158, \mathrm{~S} \\
\text { 181.972, P 213.618 }\end{array}$ & $\begin{array}{l}{ }^{24} \mathrm{Mg},{ }^{25} \mathrm{Mg},{ }^{26} \mathrm{Mg} \\
{ }^{28} \mathrm{Si},{ }^{29} \mathrm{Si},{ }^{30} \mathrm{Si} \\
\text { medium mass resolution mode: } \Delta \mathrm{m} / \mathrm{m} \\
(5 \% / 95 \% \text { intensity limits): }>5000\end{array}$ \\
\hline
\end{tabular}

Table S1: Instrument settings for concentration and silicon isotope ratio measurements. 
ERM-CD281

\begin{tabular}{l|l|l|l}
\hline $\boldsymbol{\delta}^{\mathbf{2 9}} \mathbf{S i} /{ }^{\mathbf{2 8}} \mathbf{S i}$ & $\mathbf{2} \mathbf{s}$ & $\boldsymbol{\delta}^{\mathbf{3 0}} \mathbf{S i}{ }^{\mathbf{2 8}} \mathbf{S i}$ & $\mathbf{2} \mathbf{~}$ \\
\hline-0.12 & 0.04 & -0.25 & 0.05 \\
\hline-0.18 & 0.05 & -0.33 & 0.06 \\
\hline-0.16 & 0.04 & -0.26 & 0.05 \\
\hline-0.19 & 0.05 & -0.24 & 0.07 \\
\hline-0.15 & 0.05 & -0.27 & 0.05 \\
\hline-0.19 & 0.05 & -0.28 & 0.07 \\
\hline-0.15 & 0.06 & -0.18 & 0.07 \\
\hline-0.25 & 0.04 & -0.45 & 0.05 \\
\hline-0.26 & 0.04 & -0.47 & 0.05 \\
\hline-0.28 & 0.04 & -0.44 & 0.07 \\
\hline-0.27 & 0.05 & -0.46 & 0.07 \\
\hline-0.31 & 0.04 & -0.42 & 0.07 \\
\hline-0.25 & 0.04 & -0.38 & 0.07 \\
\hline
\end{tabular}

Table S2: Individually repeated analysis of BHVO-2 and ERM-CD281 for their silicon isotope composition.

BHVO-2

\begin{tabular}{|c|c|c|c|}
\hline$\delta^{29} \mathrm{Si} /{ }^{28} \mathrm{Si}$ & $2 \mathrm{~s}$ & $\delta^{30} \mathrm{Si} /{ }^{28} \mathrm{Si}$ & $2 \mathrm{~s}$ \\
\hline-0.14 & 0.06 & -0.26 & 0.07 \\
\hline-0.13 & 0.04 & -0.24 & 0.07 \\
\hline-0.18 & 0.04 & -0.30 & 0.06 \\
\hline-0.18 & 0.04 & -0.32 & 0.06 \\
\hline-0.22 & 0.05 & -0.35 & 0.07 \\
\hline-0.15 & 0.05 & -0.29 & 0.06 \\
\hline-0.27 & 0.14 & -0.40 & 0.15 \\
\hline-0.07 & 0.08 & -0.25 & 0.09 \\
\hline-0.17 & 0.05 & -0.24 & 0.07 \\
\hline-0.11 & 0.05 & -0.27 & 0.07 \\
\hline-0.16 & 0.05 & -0.26 & 0.07 \\
\hline-0.11 & 0.08 & -0.23 & 0.09 \\
\hline-0.14 & 0.06 & -0.27 & 0.10 \\
\hline-0.14 & 0.04 & -0.30 & 0.06 \\
\hline-0.17 & 0.04 & -0.29 & 0.06 \\
\hline-0.17 & 0.04 & -0.29 & 0.06 \\
\hline-0.18 & 0.05 & -0.32 & 0.07 \\
\hline-0.14 & 0.05 & -0.22 & 0.07 \\
\hline-0.19 & 0.04 & -0.35 & 0.06 \\
\hline-0.18 & 0.05 & -0.31 & 0.07 \\
\hline-0.15 & 0.05 & -0.29 & 0.06 \\
\hline-0.13 & 0.05 & -0.29 & 0.06 \\
\hline-0.16 & 0.06 & -0.26 & 0.07 \\
\hline-0.22 & 0.05 & -0.32 & 0.07 \\
\hline-0.21 & 0.05 & -0.21 & 0.07 \\
\hline-0.16 & 0.04 & -0.29 & 0.05 \\
\hline-0.16 & 0.04 & -0.33 & 0.05 \\
\hline-0.20 & 0.04 & -0.31 & 0.06 \\
\hline-0.13 & 0.06 & -0.25 & 0.09 \\
\hline-0.16 & 0.05 & -0.35 & 0.06 \\
\hline-0.17 & 0.04 & -0.24 & 0.07 \\
\hline-0.18 & 0.05 & -0.32 & 0.07 \\
\hline-0.18 & 0.05 & -0.30 & 0.07 \\
\hline-0.17 & 0.05 & -0.36 & 0.07 \\
\hline-0.13 & 0.04 & -0.25 & 0.05 \\
\hline-0.12 & 0.04 & -0.29 & 0.04 \\
\hline-0.15 & 0.06 & -0.29 & 0.06 \\
\hline-0.09 & 0.04 & -0.26 & 0.07 \\
\hline-0.16 & 0.04 & -0.27 & 0.05 \\
\hline-0.15 & 0.04 & -0.23 & 0.06 \\
\hline
\end{tabular}




\begin{tabular}{|c|c|c|c|c|c|c|c|c|c|c|}
\hline & & $\mathbf{C a}$ & $\mathbf{F e}$ & $\mathbf{K}$ & Mg & $\mathbf{P}$ & $\mathbf{S}$ & $\mathbf{S i}$ & $\delta^{30} \mathrm{Si}$ & 2 SD \\
\hline & & $\mu \mathrm{g} / \mathrm{g}$ & $\mu \mathrm{g} / \mathrm{g}$ & $\mu \mathrm{g} / \mathrm{g}$ & $\mu \mathrm{g} / \mathrm{g}$ & $\mu \mathrm{g} / \mathrm{g}$ & $\mu \mathrm{g} / \mathrm{g}$ & $\mu \mathrm{g} / \mathrm{g}$ & $\%$ & \\
\hline \multirow{3}{*}{ Mustard } & Pot 1 & 64.2 & 4.6 & 210.8 & 14.3 & 20.4 & 21.2 & 49.3 & -0.23 & 0.12 \\
\hline & Pot 4 & 64.3 & 4.6 & 214.8 & 14.3 & 20.9 & 21.6 & 49.8 & -0.19 & 0.06 \\
\hline & Pot 7 & 65.0 & 4.7 & 216.1 & 14.4 & 21.2 & 21.6 & 49.5 & -0.15 & 0.06 \\
\hline \multirow{3}{*}{$\begin{array}{l}\text { Spring } \\
\text { Wheat }\end{array}$} & Pot 2 & 64.3 & 4.6 & 213.2 & 14.3 & 20.7 & 21.3 & 49.9 & -0.18 & 0.03 \\
\hline & Pot 5 & 64.5 & 4.7 & 214.0 & 14.3 & 20.8 & 21.6 & 49.4 & -0.18 & 0.13 \\
\hline & Pot 8 & 64.8 & 4.6 & 215.5 & 14.2 & 21.0 & 21.7 & 49.2 & -0.24 & 0.07 \\
\hline \multirow{3}{*}{ Tomato } & Pot 3 & 64.9 & 4.7 & 213.3 & 14.4 & 20.9 & 21.7 & 49.4 & -0.20 & 0.08 \\
\hline & Pot 6 & 64.5 & 4.7 & 215.4 & 14.4 & 21.1 & 21.6 & 49.5 & -0.25 & 0.10 \\
\hline & Pot 9 & 64.7 & 4.7 & 214.7 & 14.2 & 21.1 & 21.7 & 49.4 & -0.23 & 0.02 \\
\hline Average & & & & & & & & & -0.21 & 0.07 \\
\hline
\end{tabular}

Table S3: Starting composition (major element concentration (in $\mu \mathrm{g} g-1$ ) and silicon isotopic composition) of the nutrient solutions for the individual pots.

The Table S4 is in on the following pages.

25 Table S4: Dry weight, major element concentration (in $\mathrm{mg}^{-1} \mathrm{~g}^{-1}$ ) and Si isotope composition (in \%o) of the plants separated into shoot and root. 


\begin{tabular}{|c|c|c|c|c|c|c|c|c|c|c|c|c|c|c|c|}
\hline & & & dry mass & $\mathrm{Ca}$ & $\mathrm{Fe}$ & $\mathrm{K}$ & $\mathrm{Mg}$ & $\mathrm{P}$ & $\mathrm{s}$ & $\mathrm{Si}$ & & ${ }^{30} \mathrm{Si}$ & & ${ }^{30} \mathrm{Si}$ & \\
\hline \multicolumn{2}{|c|}{ Mustard } & Plant ID & [g] & $\mathrm{mg} / \mathrm{g}$ & $\mathrm{mg} / \mathrm{g}$ & $\mathrm{mg} / \mathrm{g}$ & $\mathrm{mg} / \mathrm{g}$ & $\mathrm{mg} / \mathrm{g}$ & $\mathrm{mg} / \mathrm{g}$ & $\mathrm{mg} / \mathrm{g}$ & \% NBS28 & 2 s / $* 95 \% \mathrm{Cl}$ & $\begin{array}{l}\text { \%o nutrient } \\
\text { solution }\end{array}$ & 2 s / *95 \% Cl & \\
\hline \multirow{8}{*}{ Pot 1} & \multirow{4}{*}{ Roots } & 19-5-1R-S1 & 0.96 & 3.9 & 1.0 & 23.3 & 2.6 & 5.9 & 4.0 & 13.2 & -0.83 & 0.17 & -0.62 & 0.17 & \\
\hline & & 19-5-1R-S2 & 1.48 & 2.2 & 0.5 & 29.6 & 1.9 & 4.6 & 3.3 & 11.2 & -1.15 & 0.05 & -0.94 & 0.05 & \\
\hline & & 19-5-1R-S3 & 0.58 & 2.3 & 0.5 & 27.7 & 1.2 & 4.5 & 3.3 & 11.3 & -0.92 & 0.05 & -0.72 & 0.05 & \\
\hline & & 19-5-1R-S4 & 0.05 & 4.2 & 0.9 & 15.2 & 0.9 & 3.2 & 2.2 & 5.0 & -0.73 & 0.12 & -0.53 & 0.12 & \\
\hline & \multirow{4}{*}{ Shoot } & 19-5-1S-S1 & 4.12 & 13.5 & 0.10 & 51.4 & 3.7 & 4.3 & 4.6 & 0.90 & -0.27 & 0.08 & -0.06 & 0.08 & \\
\hline & & $19-5-1 S-S 2$ & 12.12 & 18.7 & 0.07 & 46.0 & 3.7 & 5.1 & 3.7 & 1.12 & -0.38 & 0.04 & -0.18 & 0.04 & \\
\hline & & 19-5-1S-S3 & 4.43 & 20.5 & 0.06 & 44.9 & 3.0 & 5.0 & 3.6 & 0.90 & -0.50 & 0.08 & -0.29 & 0.08 & \\
\hline & & 19-5-1S-S4 & 0.63 & 18.4 & 0.15 & 53.1 & 3.0 & 8.1 & 7.4 & 0.89 & -0.38 & 0.08 & -0.17 & 0.08 & \\
\hline \multirow{8}{*}{ Pot 4} & \multirow{4}{*}{ Roots } & 19-5-4R-S1 & 0.71 & 3.3 & 0.67 & 15.2 & 1.8 & 3.2 & 3.7 & 7.23 & -0.59 & 0.04 & -0.39 & 0.04 & \\
\hline & & 19-5-4R-S2 & 0.62 & 2.7 & 0.37 & 15.5 & 1.6 & 4.4 & 2.8 & 4.75 & -1.26 & 0.08 & -1.06 & 0.08 & \\
\hline & & 19-5-4R-S3 & 0.61 & 2.6 & 0.40 & 17.8 & 1.8 & 4.1 & 1.6 & 9.00 & -1.32 & 0.07 & -1.11 & 0.07 & \\
\hline & & 19-5-4R-S4 & 1.58 & 2.1 & 0.38 & 18.1 & 1.6 & 4.1 & 2.1 & 8.19 & -0.82 & 0.04 & -0.62 & 0.04 & \\
\hline & \multirow{4}{*}{ Shoot } & 19-5-4S-S1 & 5.84 & 34.1 & 0.10 & 50.9 & 5.1 & 4.0 & 4.4 & 1.68 & -0.32 & 0.11 & -0.11 & 0.11 & \\
\hline & & 19-5-4S-S2 & 3.08 & 12.4 & 0.12 & 47.1 & 3.7 & 5.7 & 4.2 & 0.95 & -0.25 & 0.18 & -0.05 & 0.18 & \\
\hline & & 19-5-4S-S3 & 4.83 & 11.0 & 0.08 & 44.4 & 3.3 & 4.9 & 2.2 & 1.11 & -0.33 & 0.05 & -0.12 & 0.05 & \\
\hline & & $19-5-4 S-S 4$ & 10.54 & 9.0 & 0.08 & 47.5 & 3.0 & 5.3 & 2.8 & 1.04 & -0.18 & 0.08 & 0.02 & 0.08 & \\
\hline \multirow{8}{*}{ Pot 7} & \multirow{4}{*}{ Roots } & 19-5-7R-S1 & 1.98 & 1.6 & 0.21 & 18.5 & 1.6 & 3.7 & 2.3 & 3.08 & -1.19 & 0.07 & -0.99 & 0.07 & \\
\hline & & 19-5-7R-S2 & 0.22 & 2.7 & 0.89 & 24.1 & 1.2 & 3.1 & 3.5 & 17.95 & -0.74 & 0.07 & -0.54 & 0.07 & \\
\hline & & 19-5-7R-S3 & 0.80 & 2.0 & 0.16 & 21.2 & 0.9 & 3.1 & 3.4 & 3.85 & -1.05 & 0.10 & -0.84 & 0.10 & \\
\hline & & 19-5-7R-S4 & 2.15 & 1.4 & 0.19 & 24.8 & 1.1 & 3.2 & 2.8 & 8.07 & -1.07 & 0.09 & -0.86 & 0.09 & \\
\hline & \multirow{4}{*}{ Shoot } & 19-5-7S-S1 & 8.90 & 6.9 & 0.08 & 37.7 & 2.0 & 3.1 & 1.6 & 0.53 & -0.07 & 0.09 & 0.13 & 0.09 & \\
\hline & & $19-5-7 S-S 2$ & 0.93 & 4.6 & 0.06 & 44.2 & 1.9 & 2.5 & 1.7 & 0.52 & -0.04 & 0.10 & 0.17 & 0.10 & \\
\hline & & $19-5-7 S-S 3$ & 5.30 & 17.8 & 0.06 & 48.4 & 2.5 & 4.7 & 3.2 & 0.77 & -0.38 & 0.09 & -0.18 & 0.09 & \\
\hline & & 19-5-7S-S4 & 14.40 & 12.7 & 0.06 & 52.4 & 2.3 & 3.0 & 1.8 & 1.25 & 0.07 & 0.06 & 0.28 & 0.06 & \\
\hline \multirow{2}{*}{ Average } & Roots & & 0.98 & 2.59 & 0.51 & 20.90 & 1.52 & 3.92 & 2.92 & 8.57 & -0.97 & 0.15 & -0.77 & 0.15 & * \\
\hline & Shoot & & 6.26 & 14.96 & 0.08 & 47.34 & 3.10 & 4.63 & 3.44 & 0.97 & -0.25 & 0.11 & -0.05 & 0.11 & $*$ \\
\hline Sum & Plants & & 86.88 & & & & & & & & & & & & \\
\hline
\end{tabular}




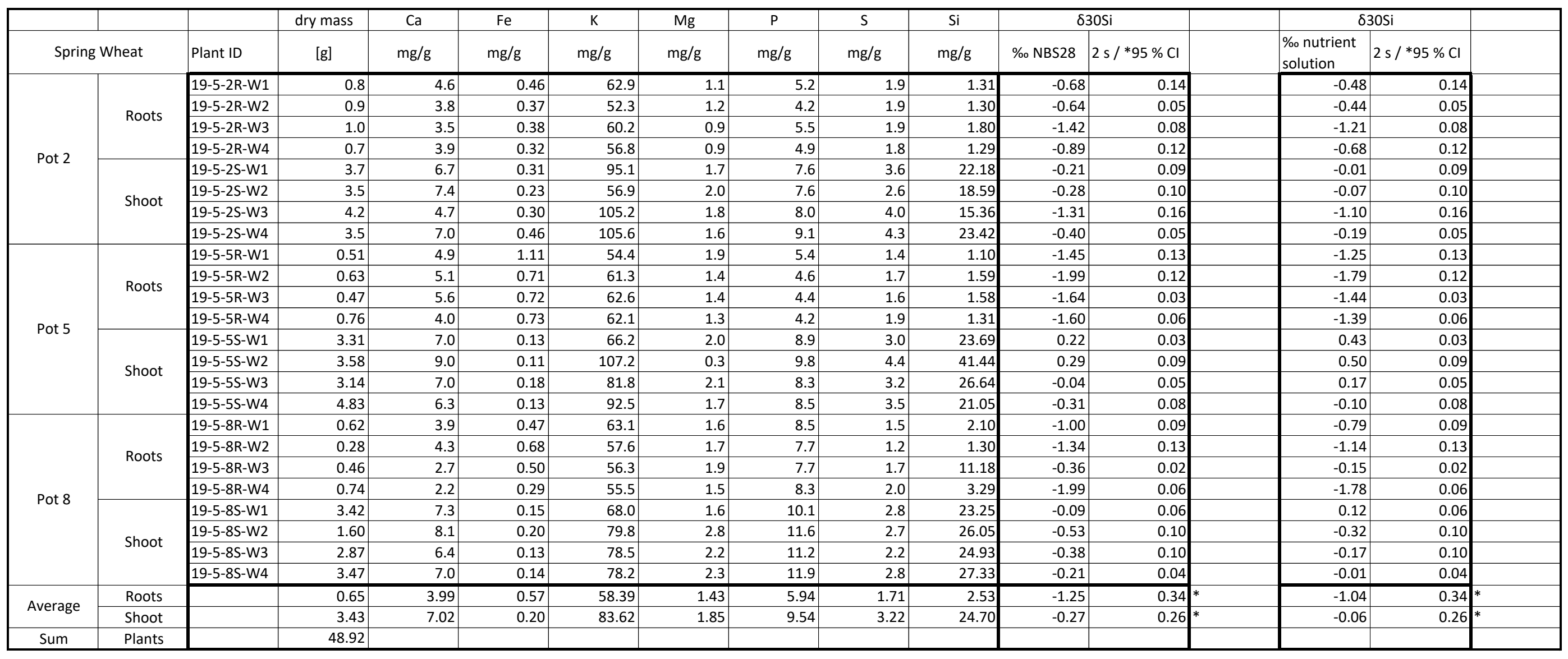




\begin{tabular}{|c|c|c|c|c|c|c|c|c|c|c|c|c|c|c|c|}
\hline & & & dry mass & $\mathrm{Ca}$ & $\mathrm{Fe}$ & K & $\mathrm{Mg}$ & $\mathrm{P}$ & $\mathrm{S}$ & $\mathrm{Si}$ & & $30 \mathrm{Si}$ & & $30 \mathrm{Si}$ & \\
\hline & & Plant ID & [g] & $\mathrm{mg} / \mathrm{g}$ & $\mathrm{mg} / \mathrm{g}$ & $\mathrm{mg} / \mathrm{g}$ & $\mathrm{mg} / \mathrm{g}$ & $\mathrm{mg} / \mathrm{g}$ & $\mathrm{mg} / \mathrm{g}$ & $\mathrm{mg} / \mathrm{g}$ & \%o NBS28 & $2 \mathrm{~s} / * 95 \% \mathrm{Cl}$ & $\begin{array}{l}\begin{array}{l}\% \text { nutrient } \\
\text { solution }\end{array} \\
\end{array}$ & $2 \mathrm{~s} / * 95 \% \mathrm{Cl}$ & \\
\hline & & 19-5-3R-T1 & 0.03 & 8.7 & 1.53 & 65.4 & 4.6 & 6.1 & 5.7 & 7.41 & -0.60 & 0.10 & \begin{tabular}{|r|}
-0.39 \\
\end{tabular} & 0.10 & \\
\hline & & 19-5-3R-T2 & 1.08 & 7.3 & 0.99 & 81.1 & 3.8 & 7.5 & 2.7 & 1.77 & -0.03 & 0.04 & 0.17 & 0.04 & \\
\hline & Roots & 19-5-3R-T3 & 0.40 & 6.8 & 1.18 & 58.5 & 4.6 & 7.6 & 2.6 & 4.32 & -0.46 & 0.03 & -0.25 & 0.03 & \\
\hline Pot 3 & & 19-5-3R-T4 & 0.44 & 6.8 & 2.48 & 72.5 & 3.4 & 9.1 & 2.8 & 3.80 & -0.19 & 0.01 & 0.02 & 0.01 & \\
\hline Pot 3 & & 19-5-3S-T1 & 0.17 & 19.5 & 0.11 & 79.8 & 3.2 & 11.5 & 2.9 & 2.45 & -0.30 & 0.09 & -0.10 & 0.09 & \\
\hline & & 19-5-3S-T2 & 6.18 & 24.9 & 0.13 & 60.2 & 3.1 & 7.9 & 4.6 & 1.21 & -0.77 & 0.01 & -0.56 & 0.01 & \\
\hline & Shoot & 19-5-3S-T3 & 2.13 & 28.9 & 0.28 & 50.7 & 5.5 & 10.9 & 4.8 & 1.38 & -0.42 & 0.09 & -0.21 & 0.09 & \\
\hline & & 19-5-3S-T4 & 3.33 & 21.5 & 0.15 & 70.1 & 3.9 & 8.3 & 4.6 & 1.23 & -0.81 & 0.06 & -0.60 & 0.06 & \\
\hline & & 19-5-6R-T1 & 0.45 & 6.0 & 1.53 & 58.7 & 4.4 & 10.0 & 3.1 & 1.90 & 0.07 & 0.10 & 0.28 & 0.10 & \\
\hline & Bonts & 19-5-6R-T2 & 0.21 & 8.6 & 3.13 & 62.7 & 5.6 & 13.1 & 3.8 & 4.12 & -0.47 & 0.10 & -0.27 & 0.10 & \\
\hline & Roots & 19-5-6R-T3 & 0.45 & 6.4 & 2.63 & 68.7 & 3.9 & 12.2 & 2.3 & 1.79 & 0.22 & 0.10 & 0.43 & 0.10 & \\
\hline Pot 6 & & 19-5-6R-T4 & 0.69 & 6.7 & 1.22 & 76.4 & 4.7 & 10.6 & 2.8 & 1.92 & -0.15 & 0.07 & 0.06 & 0.07 & \\
\hline Pot & & 19-5-6S-T1 & 2.62 & 14.8 & 0.14 & 53.9 & 4.6 & 8.0 & 4.6 & 0.89 & -0.57 & 0.11 & -0.37 & 0.11 & \\
\hline & Shoot & 19-5-6S-T2 & 1.40 & 25.9 & 0.23 & 61.6 & 5.1 & 11.7 & 6.2 & 0.98 & -0.36 & 0.14 & -0.15 & 0.14 & \\
\hline & Shoot & 19-5-6S-T3 & 2.52 & 24.3 & 0.16 & 62.6 & 4.8 & 11.7 & 5.1 & 1.63 & -0.70 & 0.12 & -0.49 & 0.12 & \\
\hline & & 19-5-6S-T4 & 3.64 & 25.6 & 0.15 & 60.1 & 4.3 & 10.0 & 4.5 & 1.01 & -0.67 & 0.11 & -0.47 & 0.11 & \\
\hline & & 19-5-9R-T1 & 0.02 & 9.4 & 1.75 & 99.0 & 3.1 & 9.5 & 9.2 & 4.65 & -0.35 & 0.07 & -0.14 & 0.07 & \\
\hline & Ronts & 19-5-9R-T2 & 0.05 & 6.8 & 1.99 & 78.2 & 5.5 & 15.2 & 3.9 & 3.10 & -0.34 & 0.15 & -0.14 & 0.15 & \\
\hline & Roots & 19-5-9R-T3 & 0.10 & 8.1 & 2.82 & 76.8 & 5.3 & 13.0 & 5.0 & 5.10 & -0.14 & 0.04 & 0.07 & 0.04 & \\
\hline & & 19-5-9R-T4 & 1.30 & 6.6 & 0.70 & 81.4 & 3.2 & 9.8 & 2.8 & 1.71 & 0.12 & 0.12 & 0.32 & 0.12 & \\
\hline Pot 9 & & 19-5-9S-T1 & 0.21 & 27.6 & 0.18 & 80.1 & 3.8 & 15.3 & 4.7 & 3.16 & -0.33 & 0.12 & -0.12 & 0.12 & \\
\hline & & 19-5-9S-T2 & 0.44 & 20.2 & 0.27 & 63.9 & 4.8 & 9.9 & 5.0 & 0.95 & -0.43 & 0.15 & -0.23 & 0.15 & \\
\hline & Shoot & 19-5-9S-T3 & 0.80 & 21.7 & 0.16 & 70.9 & 4.1 & 8.9 & 4.3 & 0.99 & -0.61 & 0.05 & -0.41 & 0.05 & \\
\hline & & 19-5-9S-T4 & 7.41 & 24.3 & 0.11 & 60.9 & 2.9 & 7.1 & 3.6 & 0.78 & -0.82 & 0.08 & -0.61 & 0.08 & \\
\hline & Roots & & 0.44 & 7.35 & 1.83 & 73.27 & 4.34 & 10.29 & 3.88 & 3.47 & -0.19 & 0.16 & 0.01 & 0.16 & * \\
\hline Average & Shoot & & 2.57 & 23.25 & 0.17 & 64.56 & 4.17 & 10.10 & 4.59 & 1.39 & -0.57 & 0.12 & -0.36 & 0.12 & $*$ \\
\hline Sum & Plants & & 36.09 & & & & & & & & & & & & \\
\hline * Uncertantiy & on $95 \% \mathrm{Cl}$ & & & & & & & & & & & & & & \\
\hline
\end{tabular}




\section{Methods}

\section{Method S1 Preparation of the nutrient solution}

30 The nutrient solution was prepared from technical graded salts and dissolved in $10 \mathrm{~L}$ of ultrapure water. Macro nutrients $1.23 \mathrm{~g}$ $\mathrm{MgSO}_{4} \cdot 7 \mathrm{H}_{2} \mathrm{O}, 3.54 \mathrm{~g} \mathrm{Ca}\left(\mathrm{NO}_{3}\right)_{2} \cdot 4 \mathrm{H}_{2} \mathrm{O}, 0.33 \mathrm{~g}$ Ferric sodium EDTA, $3.6 \mathrm{~g} \mathrm{KNO} 3,1.1 \mathrm{~g} \mathrm{KCl}$ and $0.82 \mathrm{~g} \mathrm{KH}_{2} \mathrm{PO}_{4}$. Micro nutrients: $0.55 \mathrm{mg} \mathrm{Al}{ }_{2}\left(\mathrm{SO}_{4}\right)_{3}, 0.28 \mathrm{mg} \mathrm{KJ}, 0.28 \mathrm{mg} \mathrm{KBr}, 0.55 \mathrm{mg} \mathrm{TiO}{ }_{2}, 0.28 \mathrm{mg} \mathrm{SnCl}{ }_{2} 2 \mathrm{H}_{2} \mathrm{O}, 0.28 \mathrm{mg} \mathrm{LiCl}, 0.39 \mathrm{mg} \mathrm{MnCl}_{2}$ $4 \mathrm{H}_{2} \mathrm{O}, 6.1 \mathrm{mg} \mathrm{H}_{3} \mathrm{BO}_{3}, 0.55 \mathrm{mg} \mathrm{ZnSO}_{4}, 0.55 \mathrm{mg} \mathrm{CuSO}_{4} 5 \mathrm{H}_{2} \mathrm{O}, 0.55 \mathrm{mg} \mathrm{NiSO}_{4} 6 \mathrm{H}_{2} \mathrm{O}, 0.55 \mathrm{mg} \mathrm{Co}\left(\mathrm{NO}_{3}\right)_{2} 6 \mathrm{H}_{2} \mathrm{O}, 0.05 \mathrm{mg} \mathrm{As} 2 \mathrm{O}_{3}$, $0.28 \mathrm{mg} \mathrm{BaCl} 2,0.05 \mathrm{mg} \mathrm{Bi}\left(\mathrm{NO}_{3}\right)_{3}, 0.05 \mathrm{mg} \mathrm{Rb}_{2} \mathrm{SO}_{4}, 0.28 \mathrm{mg} \mathrm{K}_{2} \mathrm{CrO}_{4}, 0.05 \mathrm{mg} \mathrm{KF}, 0.05 \mathrm{mg} \mathrm{PbCl}, 0.05 \mathrm{mg} \mathrm{HgCl}, 0.28 \mathrm{mg}$

$35 \mathrm{MoO}_{3}, 0.05 \mathrm{mg} \mathrm{H}_{2} \mathrm{SeO}_{4}, 0.28 \mathrm{mg} \mathrm{SrSO}_{4}, 0.05 \mathrm{mg} \mathrm{H}_{2} \mathrm{WO}_{4}, 0.05 \mathrm{mg} \mathrm{VCl}_{2}$ ). Silicon: $2.03 \mathrm{~g} \mathrm{NaSiO}_{4}$. $\mathrm{pH}$ was adjusted to 6.0 using $\mathrm{HNO}_{3}$ (PA grade).

\section{Method S2 Plant germination and growth conditions}

Plant seeds were germinated in in Petri dishes containing a nutrient solution of half the concentration than the solution used for growth experiments (Methods S1) and in the absence of $\mathrm{NaSiO}_{4}$. After cotyledons germinated, seeds and roots were 40 clamped in a foam block ( $3 \mathrm{~cm}$ high with a diameter of $2.5 \mathrm{~cm}$ ) and each seedling (foam block) transferred to a PP vial (50 $\mathrm{mL}$ centrifuge tube) filled with half-concentrated nutrient solution without $\mathrm{NaSiO}_{4}$. Two weeks later, the foam blocks including young plants were transferred to the experimental containers, four plants per container, 3 replicated container per species. These containers were opaque plastic containers $25.5 \mathrm{~cm}$ high, $20.5 \mathrm{~cm}$ deep and $20.5 \mathrm{~cm}$ wide (with a wall thickness of 0.5 $\mathrm{cm}$ ). In order to reduce evaporation and to prevent algae growth in the nutrient solution, the containers were closed with opaque

45 lids which had holes for the plants (foam blocks). Germination and plant cultivation were performed in a growth chamber under controlled conditions. The temperature in the growth chamber during the day and night was maintained at $18{ }^{\circ} \mathrm{C}$ for $14 \mathrm{~h}$ and at $15{ }^{\circ} \mathrm{C}$ for $10 \mathrm{~h}$, respectively, and the daylight intensity at the top of the container was adjusted to $350 \mu \mathrm{E} \mathrm{m}^{-2} \mathrm{~s}^{-1}$ ) at the start of the experiment. The relative humidity was maintained at approximately $65 \%$. For comparability, the cultivation conditions for the three species were the same, knowingly they are not equally suited for all species. The relatively low

50 temperatures may have inhibited the growth of the more thermophilic tomato, while the conditions for mustard and summer wheat were close to their optimum. In order to supply the roots with oxygen, perforated PVC tubes were used to inject (approx. $6 \mathrm{~L}$ ) room air into the nutrient solution twice a day for two hours each. The transpired water was replenished weekly with ultrapure water. 
55 Method S3 Dried plant and nutrient residue digestion and chromatographic purification of Si

The crucibles containing the sample (dried down nutrient solution or charred plant material with approximately $400 \mathrm{mg} \mathrm{NaOH}$ ) were placed in a high temperature furnace at $750{ }^{\circ} \mathrm{C}$ for $15 \mathrm{~min}$. After cooling down the crucibles were cleaned externally with ultrapure water and placed in precleaned $50 \mathrm{~mL}$ PP centrifuge tubes and covered with ultrapure water for $24 \mathrm{~h}$. Thereafter, the crucibles were placed in an ultra-sonic bath for $30 \mathrm{~min}$ to facilitate the dissolution of the fusion cake. This solution \#1 was

60 decanted and collected in precleaned PP flask. The silver crucibles were then stored for $\sim 3 \mathrm{~h}$ in a $0.03 \mathrm{M} \mathrm{HCl}$ solution and this solution \#2 was combined with solution \#1 in the PP flask. Using concentrated $\mathrm{HCl}$ the $\mathrm{pH}$ was adjusted to 1.5 . If the concentration was expected to be above $60 \mu \mathrm{g} \mathrm{g}^{-1}$ additional $0.03 \mathrm{M} \mathrm{HCl}$ solution was added. 1:10-fold dilution was analysed by ICP-OES to determining the Si content. Approximately $60 \mu \mathrm{g}$ Si from are loaded onto precleaned and preconditioned columns using a cation exchange resin $(1.5 \mathrm{~mL}$, DOWEX 50WX8, Sigma-Aldrich) and eluted using $5 \mathrm{~mL}$ ultrapure water.

65 The cation exchange resin is then regenerated using $\mathrm{HCl}$ and $\mathrm{HNO}_{3}$. The $\mathrm{Si}$ yield of the fusion procedure and the column chemistry was determined in a 1:10-fold dilution by ICP-OES. 